\title{
Informed consent forms for gynecologic cancer surgery: recommendations from the Korean Society of Gynecologic Oncology
}

\author{
Ha Kyun Chang ${ }^{1, *}$, Seung-Hyuk Shim ${ }^{2, *}$, Maria Lee ${ }^{3}$, Won Moo Lee ${ }^{4}$, Kyung Jin Eoh ${ }^{5}$, Heon Jong Yoo ${ }^{6}$, \\ Mi Kyung Kim ${ }^{7}$, Min Kyu Kim ${ }^{8}$, Kwang-Beom Lee ${ }^{9}$, Kyeong A So ${ }^{10}$, Young Tae Kim ${ }^{11}$, Dae Woo Lee ${ }^{12}$, \\ Doo-Yoon Hyun ${ }^{13}$, Jong-Min Lee ${ }^{14}$
}

\begin{abstract}
Department of Obstetrics and Gynecology, ${ }^{1}$ Korea University Ansan Hospital, Korea University School of Medicine, Ansan; ${ }^{2}$ Research Institute of Medical Science, Konkuk University School of Medicine, ${ }^{3}$ Seoul National University Hospital, Seoul National University College of Medicine, ${ }^{4}$ Hanyang University Seoul Hospital, Hanyang University College of Medicine, Seoul; ${ }^{5}$ Yongin Severance Hospital, Yonsei University College of Medicine, Yongin; ${ }^{6}$ Chungnam National University Sejong Hospital, Chungnam National University College of Medicine, Sejong; ${ }^{7}$ Ewha Womans University Mokdong Hospital, Ewha Womans University College of Medicine, Seoul; ${ }^{8}$ Samsung Changwon Hospital, Sungkyunkwan University School of Medicine, Changwon; ${ }^{9}$ Gachon University Gil Medical Center, Gachon University College of Medicine, Incheon; ${ }^{10}$ Konkuk University Hospital, Konkuk University School of Medicine, ${ }^{11}$ Severance Hospital, Yonsei University College of Medicine, ${ }^{12}$ Bucheon St. Mary's Hospital, The Catholic University College of Medicine, ${ }^{13}$ Attorney at Law, SeSeung LLC, ${ }^{14}$ Department of Obstetrics and Gynecology, Kyung Hee University Hospital at Gangdong, Kyung Hee University College of Medicine, Seoul, Korea
\end{abstract}

The sociomedical environment is changing. In the traditional physician-patient relationship, the physician was authoritative and the patient was obedient. The contractual relationship featured patient consent to the physician's decision. Today, the physician must explain fully the planned medical treatment, and any alternative, to the patient, who has the right to choose her treatment after considering the benefits and side-effects. The Korean Society of Gynecologic Oncology thus decided to standardize the surgical consent forms to meet the legal requirements of modern medicine, improve patient understanding of the surgical details, and protect medical staff from legal disputes. To determine the format and content, subcommittees for each cancer type collected and reviewed all relevant articles and the current consent forms of domestic medical institutions. After several meetings, 16 basic items to be included for each type of gynecologic cancer were selected. Also, to help patients understand the surgical details, figures were included. The revised forms were legally reviewed in terms of the appropriateness of the format and content. We also developed English versions to provide adequate information for foreign patients. We hope that these efforts will promote trust between patients and physicians, and contribute to effective treatment by laying a foundation of mutual respect.

Keywords: Informed consent; Gynecologic neoplasm; Gynecologic surgery; Medical ethics; Clinical practice pattern

\section{Note}

This manuscript is published simultaneously in the Journal of Gynecologic Oncology and Obstetrics \& Gynecology Science.

Supplementary associated with this article can be found online at https://doi.org/10.5468/ogs.22900.

\section{Synopsis}

Physician must explain the planned treatment and any alternative to the patient. We developed the surgical consent
Received: 2021.12.21. Revised: 2022.02.12. Accepted: 2022.02.12. Corresponding author: Jong-Min Lee

Department of Obstetrics and Gynecology, Kyung Hee University Hospital at Gangdong, Kyung Hee University College of Medicine, 892 Dongnam-ro, Gangdong-gu, Seoul 05278, Korea

E-mail:kgo02@hanmail.net

https://orcid.org/0000-0002-0562-5443

*These authors contributed equally to this work.

Articles published in Obstet Gynecol Sci are open-access, distributed under the terms of the Creative Commons Attribution Non-Commercial License (http://creativecommons. org/licenses/by-nc/3.0/) which permits unrestricted non-commercial use, distribution, and reproduction in any medium, provided the original work is properly cited.

Copyright $\odot 2022$ Korean Society of Obstetrics and Gynecology 


\section{Obstetrics \& Gynecology Science}

Vol. 65, No. 2, 2022

forms to meet the legal requirements, improve patient understanding of surgical details, and protect medical staff from legal disputes. We expect these promote trust between patient and physician and contribute to effective treatment.

\section{Background}

The sociomedical environment is progressing rapidly. Innovative medical devices are under constant development and leadership is increasingly professional. In the past, the physician-patient relationship was based on patient trust that the physician would provide appropriate treatment; the patient complied with the physician's will [1]. Often, the physician's explanation of planned treatment was insufficient [2]. The patient often followed the physician's instructions without understanding why this was necessary. Today, however, the physician-patient relationship is increasingly based on business and law. Patients are more educated than before and of higher economic status. Medical institutions are large and specialized, national health insurance coverage is extensive, and civil consciousness has grown and become organized. Therefore, patients and their authorized persons (guardians) now demand a systematic and sufficient explanation of any planned treatment, including surgery. Also, dissatisfaction with treatment outcomes, or the development of unexpected complications, now triggers medical disputes and lawsuits [2].

The 'informed consent' first used legally in the United States in the 1950s refers to voluntary authorization after a patient or guardian receives adequate information and fully comprehends the (actual or suspected) diagnosis, the treatment risks, alternative treatments, and the medical or surgical details [3-5]. Unfortunately, however, the consent forms for gynecologic cancer surgery are not standardized, and vary widely by the level and interest of the institution and the responsible medical staff. Thus, patient expectations are not met and medical personnel are not legally protected. In addition, Korea has achieved robust economic growth, excellent medical personnel, advanced medical systems, and relatively low medical costs (compared to the United States and Europe). Before the coronavirus disease 2019 (COVID-19) pandemic, the annual proportion of foreign patients had increased rapidly [6]. However, some institutions lack English consent forms.

Thus, the Korean Society of Gynecologic Oncology (KSGO) decided to develop standard consent forms including surgical and alternative treatment options for gynecologic cancer surgery to meet the legal requirements of modern medicine, improve patient understanding of the surgical details, and protect medical staff from legal disputes. In addition, the KSGO developed English versions to provide adequate information for foreign patients.

\section{The components and the role of informed surgical consent from the viewpoint of Korean medical law}

Physicians must provide thorough and accurate explanations of all treatment-related issues and obtain voluntary authorization (consent) from patients. Each patient has a 'right of self-determination' when surgery is suggested after the physician has explained the surgery, the risks, and the sideand after-effects [4]. If written informed consent is absent, surgery may be illegal even in the absence of negligence. A signed consent form is inadequate if the patient did not receive a detailed and sufficient explanation from the physician. Therefore, it is important to fully understand and reflect the contents required by Korean medical law when developing a surgical consent form. In Korean medical law, physicians, dentists, or oriental medical physicians must explain to the patients and obtain written informed consent when they undergo surgery, transfusion, or general anesthesia that may cause serious harm to their lives. In this case, it includes 1) the diagnosis that occurs or is likely to occur to the patient, 2) the necessity, method and contents of the surgery, 3) the name of the physician, dentist, or oriental medical physician who explains or participates in the surgery, 4) the after-effects or side-effects typically expected to occur depending on the surgery, 5) the matters to be observed before and after the surgery [7]. This meets the legal requirements, improves patient compliance, prevents certain risks, adjusts patient expectations, and provides objective evidence if a legal issue arises. The explanation must be given by the physician, not nurses or clerical staff. Consent by a guardian is permitted only if the patient is unable to express an intention (perhaps because of unconsciousness or incompetence). The explanation should be made in advance; the patient must have some time to think. The explanation should be tailored to the individual intellectual level of the patient. 


\section{Obstetrics \& Gynecology Science}

Ha Kyun Chang, et al. Consent forms for gynecologic cancer surgery

\section{Development of consent forms for gynecologic cancer surgery}

\section{Committee formation}

An Audit Committee (Director: Jong-Min Lee, Secretary: Seung-Hyuk Shim) of gynecologic oncologists was formed by the KSGO (Chairman: Young Tae Kim, Secretary-General: Dae Woo Lee). The committee featured subcommittees working on the 3 major gynecologic cancers: cervical cancer (Ha Kyun Chang, Kyung Jin Eoh, Heon Jong Yoo), endometrial cancer (Maria Lee, Mi Kyung Kim, Min Kyu Kim), and ovarian cancer (Won Moo Lee, Kwang-Beom Lee, Kyeong A So).

\section{Detailed process of developing the consent forms}

Each subcommittee reviewed all relevant articles and all current, domestic informed consent forms. Formal letters requesting the current consent forms were sent to 64 medical institutions; 23 responded, and the forms were reviewed. The standard terms and conditions for informed consent (to surgery, a medical procedure, physical examination, anesthesia, and sedation) of the Korea Fair Trade Commission (amended, June 22, 2016) were reflected in the new consent forms [8]. After three face-to-face meetings (April 17, 2021,

Table 1. The basic items of the consent forms for gynecologic cancer surgery

\begin{tabular}{l}
\hline Items \\
\hline Patient general information \\
Surgery information \\
Patient health status \\
Purpose and benefits of the surgery \\
Risk factors threatening the safety of the surgery \\
Alternative treatments \\
Consequences of not having the surgery \\
Surgical approaches and the possibility of change in the procedure \\
Extent of the surgery \\
Estimated duration of the surgery \\
Possibility of changing the physician (operating surgeon) \\
Risks, complications, and management after surgery \\
Precautions after surgery \\
Other matters requiring consent \\
Other comments or details \\
Confirmation and consent of the patient to the physician's \\
explanation
\end{tabular}

May 18, 2021, and June 29, 2021), 16 basic items for all gynecological cancer forms were selected. After a further 4 face-to-face (August 3, 2021, September 1, 2021, October 6, 2021, and November 3, 2021) and two remote meetings (August 13, 2021 and September 6, 2021), the 16 basic items were formalized as shown in Table 1.

To help patients understand the surgical details, figures were included in the consent form. Drafts of the form were revised 3 times by the committee members who focused on the content, the scientific evidence, readability, visibility, and the medical law. The final forms were legally reviewed by an expert. To gather the opinions of KSGO members, a public hearing was scheduled at the 27th KSGO Autumn Symposium in 2021. The questions posed and the responses of the committee are attached as Supplementary Table 1. The opinions gathered at the public hearing were reflected in the final consent forms after discussion at an Audit Committee meeting (Supplementary Figs. 1-3). The final consent forms were translated into English (Supplementary Figs. 4-6).

\section{Content and characteristics of the surgical consent forms}

\section{Common characteristics}

1) Patient general information

Patient identification number, full name, date of birth, age, and gender.

\section{2) Surgery information}

The diagnosis, the type, approaches, extent, and expected date of the surgery. The name and specialty of the operating surgeon.

\section{3) Patient health status}

Any history of disease/injury; allergy; idiosyncrasy; diabetes; hypertension/hypotension; drug abuse; airway disorder; smoking; bleeding disorder; cardiovascular, pulmonary, or renal disease; or other problems were included, as was any current medication. Test results and patient information were collected. The options were Yes/No/Unknown; the details of any 'Yes' answers were given. 


\section{Obstetrics \& Gynecology Science}

Vol. 65 , No. 2, 2022

4) Purpose and benefits of the surgery

The basic purpose and benefits of the surgery for each type of cancer were described (please refer to Supplementary Figs. 1-6 for the surgical consent forms for each type of gynecologic cancer). In addition, an explanation of possible personalized treatment guided by genetic testing was included.

5) Risk factors threatening the safety of the surgery Known risk factors threatening the safety of the surgery, including age over 70 years, a prolonged operation time, an extensive surgery, limitation of physical activity, malnourishment, or chronic disease (hypertension, diabetes, immunological disorders) were described.

\section{6) Alternative treatments}

The advantages/disadvantages of alternative treatments (radiotherapy or chemotherapy) for each cancer type, and the possibility of alternative treatments depending on age and fertility-sparing, were described (please refer to Supplementary Figs. 1-6 for the surgical consent forms for each type of gynecologic cancer).

\section{7) Consequences of not having the surgery}

For each type of cancer, the risks of disease progression, poor quality of life, and death in the absence of surgery were described (please refer to Supplementary Figs. 1-6 for the surgical consent forms for each type of gynecologic cancer).

8) Surgical approaches and the possibility of change in the procedure

General information on the surgical approaches (laparotomy or laparoscopic/robot-assisted surgery) used to treat each type of gynecologic cancer (with figures) was included (please refer to Supplementary Figs. 1-6 for the surgical consent forms for each type of gynecologic cancer). It was explained that if it was difficult to remove the lesion safely and completely because of advanced/extensive disease, an accompanying gynecologic condition, or intra-abdominal adhesions attributable to previous surgery, the surgical approach might be changed to laparotomy although laparoscopic or robotassisted surgery had been planned.

\section{9) Extent of the surgery}

The extent of the surgery for each type of gynecologic cancer was explained (with figures) (please refer to Supplemen- tary Figs. 1-6 for the surgical consent forms for each type of gynecologic cancer). It was explained that if metastasis to organs such as the gastrointestinal or urinary system was evident during surgery, or if other organs were injured, cooperation with other departments of surgery would be required. Also, the extent of the surgery might change depending on the patient's condition during surgery. If a change to the surgical approach or extent was urgently required, given that the patient could not be informed in advance, the reason for the change and the outcome would be explained to the patient or a guardian immediately after the operation.

\section{0) Estimated duration of the surgery}

The (approximate) estimated duration of the surgery for each cancer type was described (please refer to Supplementary Figs. 1-6 for the surgical consent forms for each type of gynecologic cancer).

\section{1) Possibility of changing the physician (operating surgeon)}

It was explained that if a change in the physician (operating surgeon) was inevitable because of the patient's condition or an institutional circumstance, the reason for the change should be explained to the patient or a guardian, and written consent obtained before the operation. To prevent arbitrary changes of physicians, accepted 'inevitabilities' were specified in detail ('emergency patient treatment'; 'personal reason [illness or childbirth]'). In addition, it was explained that if a change of surgeon was urgently required because of patient condition during the operation, given that consent could not be obtained, the reason for the change and the outcome would be explained to the patient or a guardian immediately after the surgery.

12) Risks, complications and management after surgery

Possible complications and their management after surgery were described for each type of gynecologic cancer (please refer to Supplementary Figs. 1-6 for the surgical consent forms for each type of gynecologic cancer). The explanations of the complex complications were itemized. These included adjacent organ injuries, bleeding, the possibility of reoperation due to hematoma, reduced motor and sensory function, pain, thromboembolism, lower extremity edema and lymphoceles, urinary disorder, bowel obstruction, intestinal anas- 


\section{Obstetrics \& Gynecology Science}

Ha Kyun Chang, et al. Consent forms for gynecologic cancer surgery

tomotic disruption, surgical wound infection, other infections, fistula, and the complications from prolonged surgery. All were described in detail [9-14].

\section{3) Precautions after surgery}

For each cancer type, the general precautions required immediately after surgery (coughing, deep breathing, and early ambulation) were described, as were other precautions required for 1 to 2 months after surgery in terms of bathing, exercise, and sexual intercourse. All conditions that should trigger a hospital visit were described (please refer to the Supplementary Figs.1-6 for the surgical consent forms for each type of gynecologic cancer).

\section{4) Other matters requiring consent}

Patients were asked to consent to anesthesia, blood transfusion, marking the surgical site, frozen section and pathological examination, the possibility of special examination, and the use of their biological material for research (HumanDerived Materials Research).

\section{5) Other comments or details}

This emphasized that diagnosis and surgery-related questions and answers should be allowed to range freely.

16) Confirmation and consent of the patient to the physician's explanation

This made it clear that the physician must explain the benefits and side-effects of the surgery, any possible change or addition to the extent of the surgery, and any possible change in surgical approach or the entire operation. The physician must explain that student doctors (medical students) attend (participate in) surgery for educational purposes, and the patient must agree to the presence of that person. If the final signature is that of a guardian rather than the patient, the reason must be specified.

\section{Specifications by gynecologic cancer type}

\section{1) Cervical cancer}

(1) The effects and disadvantages of radiotherapy as an alternative to surgery were explained, and the possibility of adjuvant radiotherapy was explained to patients at high risk of recurrence after surgery. We also explained that chemotherapy could be concurrent in medically eligible patients; this increased the effectiveness of radiotherapy $[15,16]$.

(2) In "Surgical approaches and the possibility of change in the procedure," we reflected the results of a recent, international, prospective clinical trial of surgical approaches for cervical cancer patients: minimally invasive radical hysterectomy was associated with lower rates of disease-free survival and overall survival than abdominal radical hysterectomy in women with early-stage cervical cancer [17]. Therefore we wrote: "Laparoscopy or robot-assisted surgery may be performed by a skilled gynecologic cancer specialist in patients who can achieve oncological safety, based on the tumor size and the development of surgical skills to minimize intraperitoneal metastasis of cervical cancer. Laparoscopy or robot-assisted surgery must be performed after the patient and physician have fully discussed the advantages and disadvantages of the surgical approaches" [18,19].

(3) To reflect the recently developed surgical/anatomical concept precisely, and to ensure compatibility with the surgical manual of the Korean Gynecologic Oncology Group, the type of hysterectomy was based on the Querleu and Morrow classification $[20,21]$. We prepared figures to facilitate patient understanding. In addition, conization or radical trachelectomy for early cancer patients who wished to preserve fertility were explained using figures.

\section{2) Endometrial cancer}

(1) We explained that standard treatment for patients who are operable and do not desire fertility preservation involves a staging operation including hysterectomy (removal of both ovaries and salpinges) [22].

(2) We explained that hormone therapy could be an option on a limited basis to preserve fertility, but only if the cancer was at an early stage and exhibited specific clinicopathological characteristics [22]. Also, we explained that if a patient is in poor general condition or at a high risk of developing postoperative complications, other non-surgical treatment options can be attempted, including systemic chemotherapy and radiation therapy. However, as this is not a standard alternative treatment to surgery, the prognosis may be worse than when receiving the standard treatment including surgery.

(3) We explained that ovaries can be saved if the patient wants to preserve them considering their age and understands certain risks for recurrence even in the early stage of endometrial cancer $[23,24]$. 


\section{Obstetrics \& Gynecology Science}

Vol. 65, No. 2, 2022

(4) We explained that lymphadenectomy could feature: 1) complete lymph node dissection; 2) selective dissection of (only) grossly enlarged lymph nodes; or, 3) sentinel lymph node resection only [25-28].

5) We explained that maximal cytoreduction of any suspect metastatic lesion should be performed when treating advanced-stage endometrial cancer [29].

\section{3) Ovarian cancer}

(1) The nomenclature of ovarian cancer surgery varies; one of exploratory laparotomy, cytoreductive surgery, a debulking operation, or staging operation has been described according to the preference of the institute or physician. Thus, we allowed institutions to describe the nomenclature of the operation at their discretion.

(2) In patients with ovarian cancer, the extent of the surgery varies more than that for cervical or endometrial cancer. Therefore, we did not summarize this in the "Surgery information" section for ovarian cancer (unlike the other cancers). Instead, we provide figures showing the possible metastatic lesions to help patients understand, and recommend to explain and check the scheduled surgery according to the patient's disease status in "Extent of the surgery."

(3) We explained that if complete resection was difficult because of extensive pleural effusion or ascites, or a metastasis to the mesentery or liver parenchyma, neoadjuvant chemotherapy followed by interval debulking surgery was an alternative [30]. In addition, we explained that if a patient was inappropriate for surgery (because, for example, of a medical condition) palliative chemotherapy might relieve the symptoms but such treatment was not very effective [31].

\section{Summary}

In clinical practice, the relationship between the physician and the patient is changing from a traditionally submissive relationship in which the patient obeys the physician's authority to a mutual contractual relationship based on the physician's explanation and the patient's consent. The physician must explain the planned and alternative treatments fully, and the patient has the right to choose, after considering benefits and side-effects. Well-documented consent forms may have the advantages of providing feedback on whether treatment plan for the patients is well established and implemented in terms of adequateness and efficiency as well as the patient's right of self-determination. The effort of the KSGO to develop consent forms for gynecologic cancer surgery is just part of the continuous work of experts to meet the ethical/legal demands of both physicians and patients in the era of modern medicine. The KSGO does not seek simply to strengthen the authority of physicians or to protect physicians from medical disputes but, rather, to lead the way for the development of ethical awareness in experts who are responding to the changed spirit of the times, i.e., patientcentered medical care. However, given the differences in medical systems and environments, the nature of informed surgical consent inevitably differs among institutions. Therefore, the KSGO decided to allow all institutions to edit the forms; this will (we believe) enhance utilization. The existing forms explain only the most basic surgeries for the 3 major gynecologic cancers. In the future, informed consent forms for other treatment modalities, including chemotherapy and radiation therapy, and additional surgical methods required in various clinical situations, should be developed. It is hoped that the efforts of the KSGO will promote trust between patients and physicians and contribute to the treatment of gynecologic cancers by laying a foundation of mutual respect.

\section{Conflict of interest}

No potential conflict of interest relevant to this article was reported.

\section{Author contributions}

Conceptualization: C.H.K., S.S.H., L.M., L.W.M., E.K.J., K.M.K., K.M.K., L.K.B., S.K.A., K.Y.T., L.D.W.; Data curation: S.S.H., L.J.M.; Investigation: S.S.H., L.M., L.W.M., E.K.J., Y.H.J., K.M.K., L.K.B., S.K.A., K.Y.T., L.J.M.; Methodology: C.H.K., S.S.H., L.M., L.W.M., E.K.J., Y.H.J., K.M.K., L.K.B., S.K.A., K.Y.T., L.D.W., L.J.M.; Project administration: C.H.K., S.S.H., L.M., L.W.M., K.M.K., K.Y.T., L.J.M.; Resources: K.Y.T., H.D.Y., L.J.M.; Supervision: C.H.K., S.S.H., L.M., L.W.M., Y.H.J., K.M.K., K.M.K., L.K.B., S.K.A., K.Y.T., H.D.Y., L.D.W., L.J.M.; Validation: C.H.K., S.S.H., L.W.M., L.K.B., S.K.A., K.Y.T., H.D.Y., L.J.M.; Visualization: S.S.H.; Writing - original draft: C.H.K., S.S.H., L.W.M., L.J.M.; Writing - review \& 


\section{Obstetrics \& Gynecology Science}

Ha Kyun Chang, et al. Consent forms for gynecologic cancer surgery

editing: C.H.K., S.S.H., L.M., L.W.M., E.K.J., Y.H.J., K.M.K., K.M.K., L.K.B., S.K.A., K.Y.T., L.J.M.

\section{Acknowledgements}

The authors thank all members of the Korean Society of Gynecologic Oncology for their support.

\section{Supplementary material}

Supplementary Table 1 and Figs. 1-6 associated with this article can be found online at https://doi.org/10.5468/ ogs.22900.

\section{References}

1. Stain SC. Informed surgical consent. J Am Coll Surg 2016;222:717-8.

2. Korea Medical Dispute Mediation and Arbitration Agency. Medical dispute mediation and arbitration statistical yearbook 2020 [Internet]. Seoul (KR): Korea Medical Dispute Mediation and Arbitration Agency; c2021 [cited 2021 Jun 4]. Available from: https://www.k-medi.or.kr/ lay $1 / \mathrm{bbs} / \mathrm{S} 1 \mathrm{~T} 27 \mathrm{C}$ (6)/A/25/list.do.

3. Cocanour CS. Informed consent-It's more than a signature on a piece of paper. Am J Surg 2017;214:993-7.

4. Leclercq WK, Keulers BJ, Scheltinga MR, Spauwen PH, van der Wilt GJ. A review of surgical informed consent: past, present, and future. A quest to help patients make better decisions. World J Surg 2010;34:1406-15.

5. Sarela Al, Thomson M. Balancing law, ethics and reality in informed consent for surgery. Ann R Coll Surg Engl 2014;96:329-30.

6. Korea Health Industry Development Institute. Statistics on international patients in Korea, 2018 [Internet]. Cheongju (KR): Korea Health Industry Development Institute; c2021 [cited 2021 Jun 4]. Available from: https:// www.khidi.or.kr/board/view?linkld=48806593\&menuld $=$ MENU00085.

7. Korea Ministry of Government Legislation. Medical Service Act [Internet]. Sejong (KR): Korea Legislation Research Institute; c2021 [cited 2021 Jun 4]. Avail- able from: https://elaw.klri.re.kr/kor_service/lawView. do?hseq=53532\&lang=ENG.

8. Fair Trade Commission. [No. 10003] Standard terms of consent (surgery, procedure, test, anesthesia, sedation of consciousness) [Internet]. Sejong (KR): Fair Trade Commission; c2021 [cited 2021 Jun 4]. Available from: https://www.ftc.go.kr/www/cop/bbs/selectBoardArticle. do? key=201\&nttld=82483\&bbsld=BBSMSTR_00000000 2320\&bbsTyCode=BBST01.

9. Miskovic A, Lumb AB. Postoperative pulmonary complications. Br J Anaesth 2017;118:317-34.

10. Assaad S, Kratzert WB, Shelley B, Friedman MB, Perrino A Jr. Assessment of pulmonary edema: principles and practice. J Cardiothorac Vasc Anesth 2018;32:901-14.

11. Netea MG, Kullberg BJ, Van der Meer JW. Circulating cytokines as mediators of fever. Clin Infect Dis 2000;31 Suppl 5:S178-84.

12. Carmichael $P$, Carmichael AR. Acute renal failure in the surgical setting. ANZ J Surg 2003;73:144-53.

13. Golden D, Corbett J, Forni LG. Peri-operative renal dysfunction: prevention and management. Anaesthesia 2016;71 Suppl 1:51-7.

14. Lim W, Le Gal G, Bates SM, Righini M, Haramati LB, Lang $E$, et al. American Society of Hematology 2018 guidelines for management of venous thromboembolism: diagnosis of venous thromboembolism. Blood Adv 2018;2:3226-56.

15. Keys HM, Bundy BN, Stehman FB, Muderspach LI, Chafe $W E$, Suggs $C L 3 r d$, et al. Cisplatin, radiation, and adjuvant hysterectomy compared with radiation and adjuvant hysterectomy for bulky stage IB cervical carcinoma. N Engl J Med 1999;340:1154-61.

16. Morris M, Eifel PJ, Lu J, Grigsby PW, Levenback C, Stevens $\mathrm{RE}$, et al. Pelvic radiation with concurrent chemotherapy compared with pelvic and para-aortic radiation for high-risk cervical cancer. N Engl J Med 1999;340: 1137-43.

17. Ramirez PT, Frumovitz M, Pareja R, Lopez A, Vieira M, Ribeiro $R$, et al. Minimally invasive versus abdominal radical hysterectomy for cervical cancer. N Engl J Med 2018;379: 1895-904.

18. Kim M, Kong TW, Kim S, Kim SC, Kim YB, Kim JW, et al. Minimally invasive surgery for radical hysterectomy in women with cervical cancer: Korean Society of Gynecologic Oncology, Korean Society of Obstetrics and 


\section{Obstetrics \& Gynecology Science}

Vol. 65, No. 2, 2022

Gynecology, and Korean Society of Gynecologic Endoscopy and Minimally Invasive Surgery position statement. J Gynecol Oncol 2019;30:e104.

19. Melamed A, Margul DJ, Chen L, Keating NL, Del Carmen $M G$, Yang J, et al. Survival after minimally invasive radical hysterectomy for early-stage cervical cancer. $\mathrm{N}$ Engl J Med 2018;379:1905-14.

20. Lee $M$, Choi CH, Chun YK, Kim YH, Lee KB, Lee SW, et al. Surgical manual of the Korean Gynecologic Oncology Group: classification of hysterectomy and lymphadenectomy. J Gynecol Oncol 2017;28:e5.

21. Querleu D, Morrow CP. Classification of radical hysterectomy. Lancet Oncol 2008;9:297-303.

22. American College of Obstetricians and Gynecologists. ACOG practice bulletin, clinical management guidelines for obstetrician-gynecologists, number 65, August 2005: management of endometrial cancer. Obstet Gynecol 2005;106:413-25.

23. Wright JD, Buck AM, Shah M, Burke WM, Schiff PB, Herzog TJ. Safety of ovarian preservation in premenopausal women with endometrial cancer. J Clin Oncol 2009;27:1214-9.

24. Lee TS, Lee JY, Kim JW, Oh S, Seong SJ, Lee JM, et al. Outcomes of ovarian preservation in a cohort of premenopausal women with early-stage endometrial cancer: a Korean Gynecologic Oncology Group study. Gynecol Oncol 2013;131:289-93.

25. Soliman PT, Frumovitz M, Spannuth W, Greer MJ, Sharma S, Schmeler KM, et al. Lymphadenectomy during endometrial cancer staging: practice patterns among gynecologic oncologists. Gynecol Oncol 2010;119:2914.

26. Todo Y, Kato H, Kaneuchi M, Watari H, Takeda M, Sakuragi N. Survival effect of para-aortic lymphadenectomy in endometrial cancer (SEPAL study): a retrospective cohort analysis. Lancet 2010;375:1165-72.

27. Holloway RW, Abu-Rustum NR, Backes FJ, Boggess JF, Gotlieb WH, Jeffrey Lowery W, et al. Sentinel lymph node mapping and staging in endometrial cancer: a Society of Gynecologic Oncology literature review with consensus recommendations. Gynecol Oncol 2017; 146:405-15.

28. Rossi EC, Kowalski LD, Scalici J, Cantrell L, Schuler K, Hanna RK, et al. A comparison of sentinel lymph node biopsy to lymphadenectomy for endometrial cancer staging (FIRES trial): a multicentre, prospective, cohort study. Lancet Oncol 2017;18:384-92.

29. Barlin JN, Puri I, Bristow RE. Cytoreductive surgery for advanced or recurrent endometrial cancer: a meta-analysis. Gynecol Oncol 2010;118:14-8.

30. Vergote I, Tropé CG, Amant F, Kristensen GB, Ehlen T, Johnson $\mathrm{N}$, et al. Neoadjuvant chemotherapy or primary surgery in stage IIIC or IV ovarian cancer. N Engl J Med 2010;363:943-53.

31. Heintz AP, Odicino F, Maisonneuve P, Quinn MA, Benedet JL, Creasman WT, et al. Carcinoma of the ovary. FIGO 26th annual report on the results of treatment in gynecological cancer. Int J Gynaecol Obstet 2006;95 Suppl 1:S161-92. 